\title{
FATOR DE CONDIÇÃO RELACIONADO A ASPECTOS REPRODUTIVOS DA PIAPARA (Leporinus obtusidens) (Characiformes: Anostomidae) COLETADAS A JUSANTE DA USINA HIDRELÉTRICA DO FUNIL, MINAS GERAIS, BRASIL
}

\author{
Viviane de Oliveira Felizardo ${ }^{1}$, Luis David Solis MURgas ${ }^{1}$, Elissandra Ulbricht WinkaleR ${ }^{2}$, \\ Gilmara Junqueira Machado Pereira ${ }^{3}$, MARiana Martins Drumond ${ }^{4}$, Estefânia de SouZa \\ ANDRADE $^{1}$ \\ ${ }^{1}$ Departamento de Medicina Veterinária, Setor de Fisiologia e Farmacologia da Universidade Federal de Lavras \\ (UFLA), Lavras, MG \\ ${ }^{2}$ Centro de Ciências Agrárias, Ambientais e Biológicas da Universidade Federal do Recôncavo da Bahia \\ (UFRB), Cruz das Almas, BA \\ ${ }^{3}$ Analista Ambiental da Usina Hidrelétrica de Funil, Lavras, MG \\ ${ }^{4}$ Departamento de Zootecnia, Setor de Fisiologia e Farmacologia da Universidade Federal de Lavras (UFLA), \\ Lavras, MG \\ Email para correspondência: elis@ufrb.edu.br
}

\section{RESUMO}

O objetivo deste trabalho foi descrever a variação sazonal do fator de condição (K) correlacionado com os índices gonadossomático (IGS) e hepatossomático (IHS) de Leporinus obtusidens (Valenciennes, 1837), coletados a jusante da Usina Hidrelétrica do Funil, e avaliar se esses índices podem indicar o período reprodutivo dessa espécie. Os animais $(\mathrm{n}=110)$ foram capturados mensalmente entre setembro/2006 e agosto/2007 e agrupados por estação do ano. O comprimento e o peso dos animais foram obtidos para cálculo de $\mathrm{K}$, o peso do fígado foi determinado para o cálculo do IHS e com o peso das gônadas foi obtido o IGS. O valor de K obtido para machos e fêmeas agrupados e somente para machos foi significativamente menor $(\mathrm{p}<0,05)$ no verão. Para as fêmeas não houve variação significativa de $\mathrm{K}$ ao longo do ano. Os valores de IHS foram significativamente maiores durante a primavera e verão para ambos os sexos, enquanto que o IGS de machos e fêmeas foram maiores $(\mathrm{p}<0,05)$ durante a primavera. Nos testes de correlação, $\mathrm{K}$ não está relacionado com o IGS nem com o IHS e não se mostrou um bom indicador do período reprodutivo da piapara. Entretanto, existe forte correlação entre IGS e IHS e, relacionados, podem ser utilizados como indicadores do período reprodutivo dessa espécie, que provavelmente ocorre na primavera.

PALAVRAS-CHAVE: peixe; reprodução; reservatório.

\section{CONDITION FACTOR RELATED TO REPRODUCTIVE ASPECTS OF (Leporinus obtusidens) (Characiformes: Anostomidae) COLLECTED AT DOWNSTREAM OF THE HIDROELECTRIC POWER STATION OF FUNIL, STATE OF MINAS GERAIS, BRAZIL}

\section{ABSTRACT}

This study aimed to describe the seasonal variation of condition factor (K) correlated with gonadossomatic (GSI) and hepatossomatic index (HSI) of Leporinus obtusidens
(Valenciennes, 1837), collected downstream of the Hidroelectric Power Station of Funil, state of Minas Gerais, Brazil, and to evaluate if these indices can indicate 
the reproductive period of this species. The animals were captured between September/2006 and August/2007 and grouped seasonally. The length and the weight of 110 individuals were measured to calculate $\mathrm{K}$, the weight of the liver was determined to calculate HSI and the weight of the gonad to calculate GSI. The $\mathrm{K}$ of females and males grouped and of males alone were significantly lower $(p<0.05)$ in the summer. As for the females alone, no significant variation of the $\mathrm{K}$ was observed throughout the

KEYWORDS: fish; reproduction; reservoir.

\section{INTRODUÇÃO}

A relação entre o peso e o comprimento corporal permite calcular um parâmetro que determina o grau de bem estar do peixe, denominado de Fator de Condição (K). Esse índice é amplamente utilizado, pois fornece importantes informações sobre o estado fisiológico do animal, a partir do pressuposto de que indivíduos com maior massa em um dado comprimento estão em melhor condição (VAZZOLER, 1996). Além de permitir comparações entre populações de peixes submetidas a diferentes condições de clima, temperatura, alimentação, densidade e qualidade da água, o cálculo do fator de condição também pode estar relacionado com os aspectos reprodutivos dos peixes (AGOSTINHO et al., 1990).

Estudos sobre a dinâmica da reprodução de peixes ocupam importante lugar na investigação pesqueira, fornecendo subsídios necessários para a elaboração de programas visando à exploração racional e preservação da ictiofauna de rios e lagos (Santos et al., 2006; Prestes et al., 2010). Dentro dessa linha de pesquisa, destacam-se estudos que abordam época de reprodução, tamanho e idade da primeira maturação gonadal, fecundidade, taxa de crescimento e tipo de desova (SANTOS et al., 2006; GOMIERO et al., 2008; GOMIERO et al., 2010).

A espécie Leporinus obtusidens (Valenciennes, 1837), popularmente conhecida como piapara, é um peixe de água doce nativo das regiões Sul e Sudeste do Brasil (NETO et al., 2006), possui hábito alimentar onívoro, alimentando-se de insetos, restos de peixes e de vegetais e é uma espécie economicamente importante no sul do Brasil (TAITSON et al., 2008). A espécie se situa nos elos intermediários da cadeia trófica, devido a seus hábitos alimentares. Por essa razão, é de grande importância na manutenção do equilíbrio do ciclo biológico dos ambientes em que habita, constituindo o principal produto de captura, principalmente no alto rio Paraná (ARAYA et al., 2005).

A piapara migra a montante, uma vez por ano, year. The values of HSI were significantly greater during the spring and summer for both sexes, whereas the GSI of males and females was superior $(\mathrm{p}<0.05)$ in the summer. In the correlation tests, $\mathrm{K}$ was not related with the GSI and HSI factors; however, strong correlation between GSI and HSI was found. $\mathrm{K}$ is not an index of the reproductive period of Leporinus obtusidens (Valenciennes, 1837), whereas GSI and HIS can be used for indicating reproduction.

para a desova (OLDANI et al., 1992). Assim como a maioria das espécies de peixes, mostra uma periodicidade em seu processo reprodutivo, iniciando seu desenvolvimento gonadal em uma época anterior àquela de reprodução e complementando sua maturação gonadal no momento em que as condições ambientais são adequadas à fecundação e ao desenvolvimento reprodutivo (VAZZOLER, 1996).

Os represamentos promovem grandes alterações nas interações bióticas dentro do ecossistema, particularmente entre as de natureza trófica e reprodutiva dos peixes, devido à interrupção das rotas migratórias impostas pelo barramento (AGOSTINHO et al., 2007). Dessa forma, devido aos sucessivos barramentos de rios, as espécies tendem a se adequar às novas situações ecológicas, para poder realizar satisfatoriamente o ciclo reprodutivo (SUZUKI \& AGOSTINHO, 1997).

Com isso, os objetivos deste trabalho foram descrever o padrão de variação sazonal do fator de condição e correlacioná-lo com os índices gonadossomático e hepatossomático e determinar se esses índices podem ser utilizados como ferramentas indicativas do período reprodutivo de $L$. obtusidens, coletados a jusante da Usina Hidrelétrica do Funil, Perdões, MG.

\section{MATERIAL E MÉTODOS}

Os exemplares de piapara foram coletados no rio Grande a jusante da barragem da Usina Hidrelétrica do Funil, situada no município de Perdões - MG (Lat $44^{\circ} 55^{\prime}$ Long $21^{\circ} 05^{\prime}$ ). Os animais foram capturados com molinete e anzol, utilizandose como isca minhoca e coração bovino. As coletas foram realizadas mensalmente, entre setembro/2006 a agosto/2007, e foram agrupadas por estação do ano: primavera (23/09 a 21/12), verão (21/12 a 21/03), outono (21/03 a 21/06) e inverno (21/06 a 23/09).

Após a captura, os peixes foram anestesiados com benzocaína (1g/10 mL álcool + 15 L de água) e identificados com etiqueta fixada na nadadeira 
dorsal. Para cada exemplar foi registrado o sexo, determinados o comprimento total (Lt) e padrão (Lp) em centímetros (com auxílio de ictiômetro) e o peso total $(\mathrm{Wt})$, peso das gônadas $(\mathrm{Wg})$ e peso do fígado (Wf) em gramas, com uso de balança digital (Shimadzu $^{\circledR}$, modelo BL), com precisão de 0,01 gramas. O período reprodutivo foi estabelecido por meio da análise sazonal do fator de condição (K) e do índice hepatossomático (IHS), comparando-os com a curva de maturação, com base na variação sazonal do índice gonadossomático (IGS).

$\mathrm{O} \mathrm{K}$ dos animais foi calculado pelo método alométrico, a partir da expressão $\mathrm{K}=\mathrm{W} / \mathrm{L}^{\mathrm{b}}$, na qual $\mathrm{W}$ representa a massa total, L o comprimento padrão dos indivíduos e b o coeficiente de regressão. Para estimar o valor do coeficiente b, ajustou-se uma única equação de relação peso-comprimento $\left(\mathrm{W}=\mathrm{a} \mathrm{L}^{\mathrm{b}}\right)$, a partir do conjunto de todos os indivíduos coletados, utilizando-se o método dos mínimos quadrados aplicados aos dados convertidos em seus respectivos logaritmos naturais, de acordo com metodologia sugerida por LIMA-JUNIOR et al. (2002).

Para a análise da variação sazonal do desenvolvimento gonadal, estimou-se o índice gonadossomático para cada exemplar, a partir da expressão IGS=(Wg/Wt)100 e, para se determinar o índice hepatossomático dos animais, utilizou-se a fórmula IHS=(Wf/Wt)100.

Os resultados obtidos para as variáveis K, IGS e IHS ao longo das quatro estações foram submetidos à comparação sazonal, pela aplicação do teste de Kruskal-Wallis, após a análise de normalidade dos dados pelo teste de Shapiro-Wilk. A possível correlação entre essas variáveis foi testada pela aplicação do Coeficiente de Correlação de Spearman. O nível de significância de 0,05 foi estabelecido para todos os tratamentos e o programa estatístico utilizado foi BioEstat 5.0.

\section{RESULTADOS}

Os indivíduos capturados $(\mathrm{n}=110)$ foram classificados em 63 fêmeas e 47 machos (Tabela 1). A amplitude de variação dos valores de comprimento padrão foi de 25 a $62 \mathrm{~cm}$, enquanto que a massa corpórea total variou de 200 a 3500 g.

Tabela 1. Número de indivíduos de cada sexo de $L$. obtusidens capturados nas diferentes estações do ano no período de setembro/2006 a agosto/2007, a jusante da UHE do Funil, no município de Perdões (MG)

\begin{tabular}{lccc}
\hline & \multicolumn{3}{c}{ Número de indivíduos } \\
& Fêmeas & Machos & Total \\
\hline Primavera (23/09 a 21/12) & 21 & 12 & 33 \\
Verão (21/12 a 21/03) & 11 & 12 & 23 \\
Outono (21/03 a 21/06) & 17 & 15 & 32 \\
Inverno (21/06 a 23/09) & 14 & 8 & 22 \\
\hline & 47 & 63 & 110 \\
\hline
\end{tabular}

A equação da relação peso-comprimento, ajustada a partir dos dados dos indivíduos capturados, assumiu a seguinte forma: $\mathrm{W}=0,019 * \mathrm{~L}^{3,142}$. Assim, o valor do coeficiente $\mathrm{b}$ determinado e aplicado na fórmula para cálculo do $\mathrm{K}$ foi de 3,142 (Figura 1).

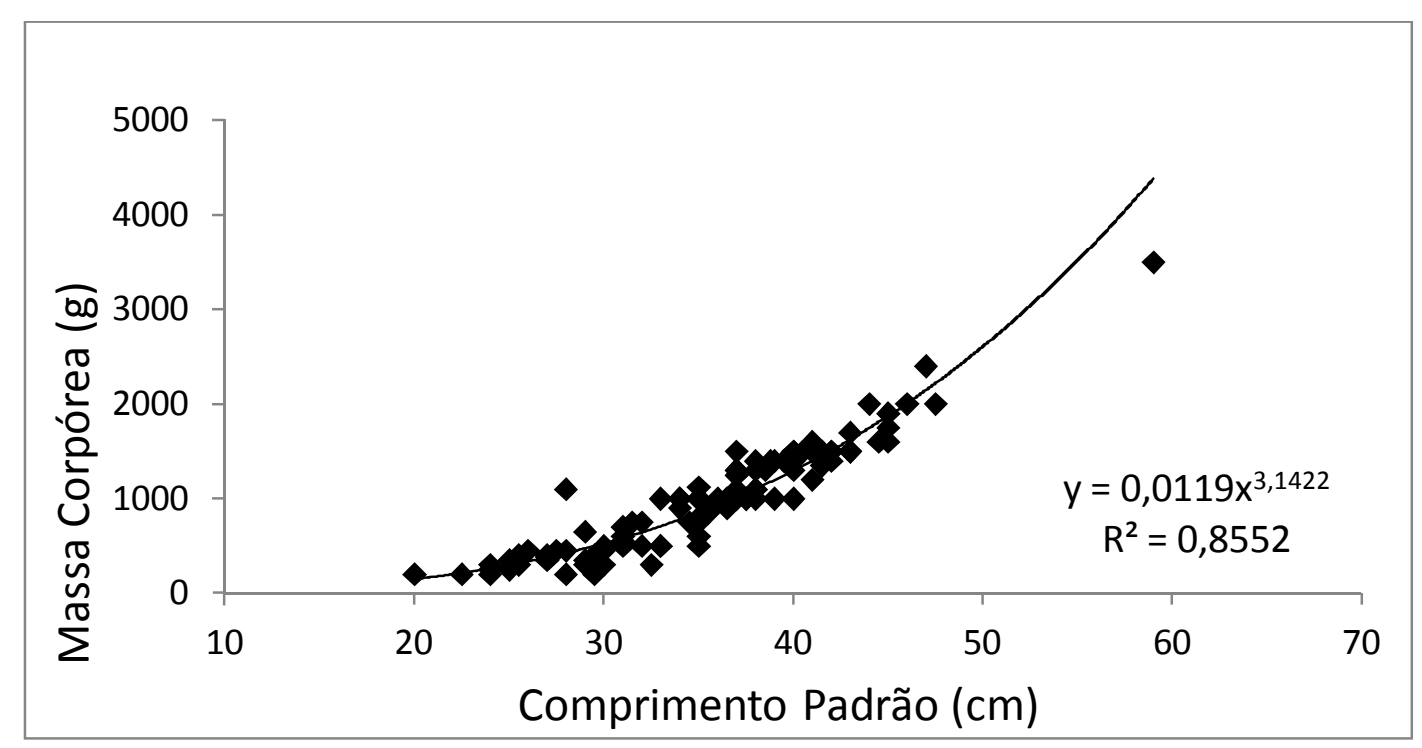

Figura 1. Diagrama de dispersão dos dados de comprimento padrão e peso total de L. obtusidens capturados nas diferentes estações do ano no período de setembro/2006 a agosto/2007, a jusante da UHE do Funil, no município de Perdões (MG). 
O fator de condição das piaparas, machos e fêmeas agrupados, foi significativamente menor no verão, quando comparado com o outono e inverno (Figura 2A). Quando analisado em relação ao sexo (Figura 2B), para as fêmeas o $\mathrm{K}$ não apresentou diferenças sazonais significativas $(p=0,1215)$, enquanto que para os machos o menor valor de $\mathrm{K}$ também foi observado no verão, em relação ao inverno.
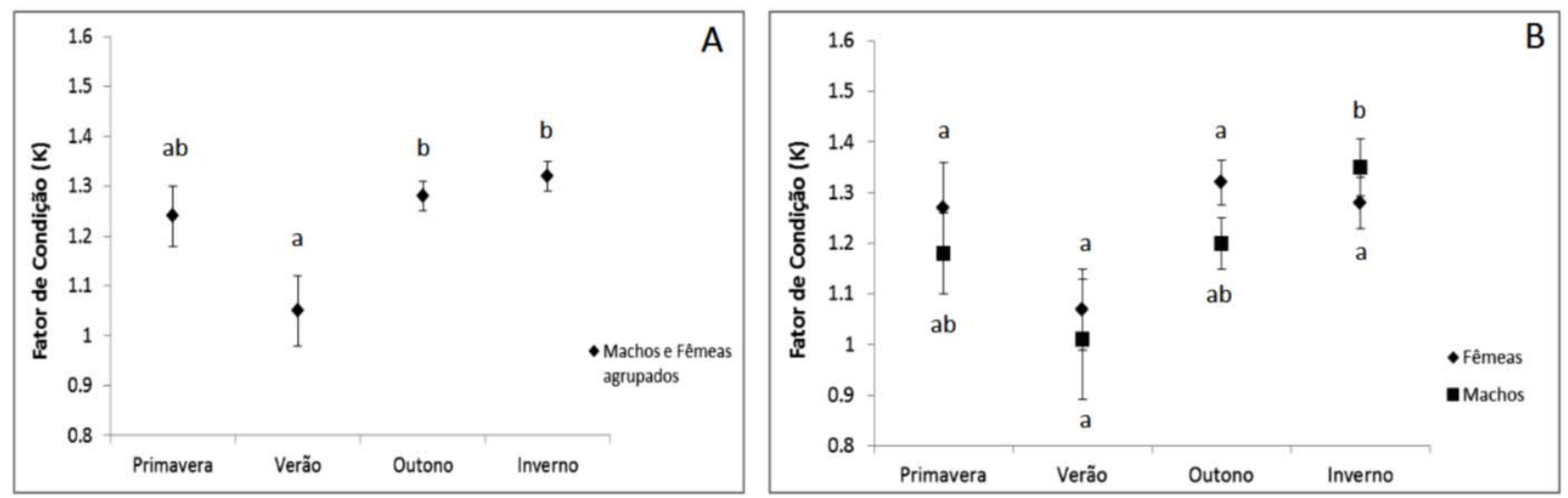

Figura 2. Médias e erro padrão do fator de condição (K) de machos e fêmeas agrupados (A) e de machos e fêmeas (B) de L. obtusidens capturadas a jusante da UHE do Funil, no município de Perdões (MG), no período de setembro/2006 a agosto/2007. Letras iguais indicam resultados significativamente iguais, letras diferentes, indicam valores significativamente diferentes, com $\mathrm{p}<0,05$.

O índice gonadossomático das piaparas fêmeas foi maior $(\mathrm{p}<0,05)$ na primavera, quando comparado com as demais estações (Figura 3A). Nos machos, o maior valor do IGS $(\mathrm{p}<0,05)$ também foi observado na primavera, em relação ao outono e inverno (Figura 3B).
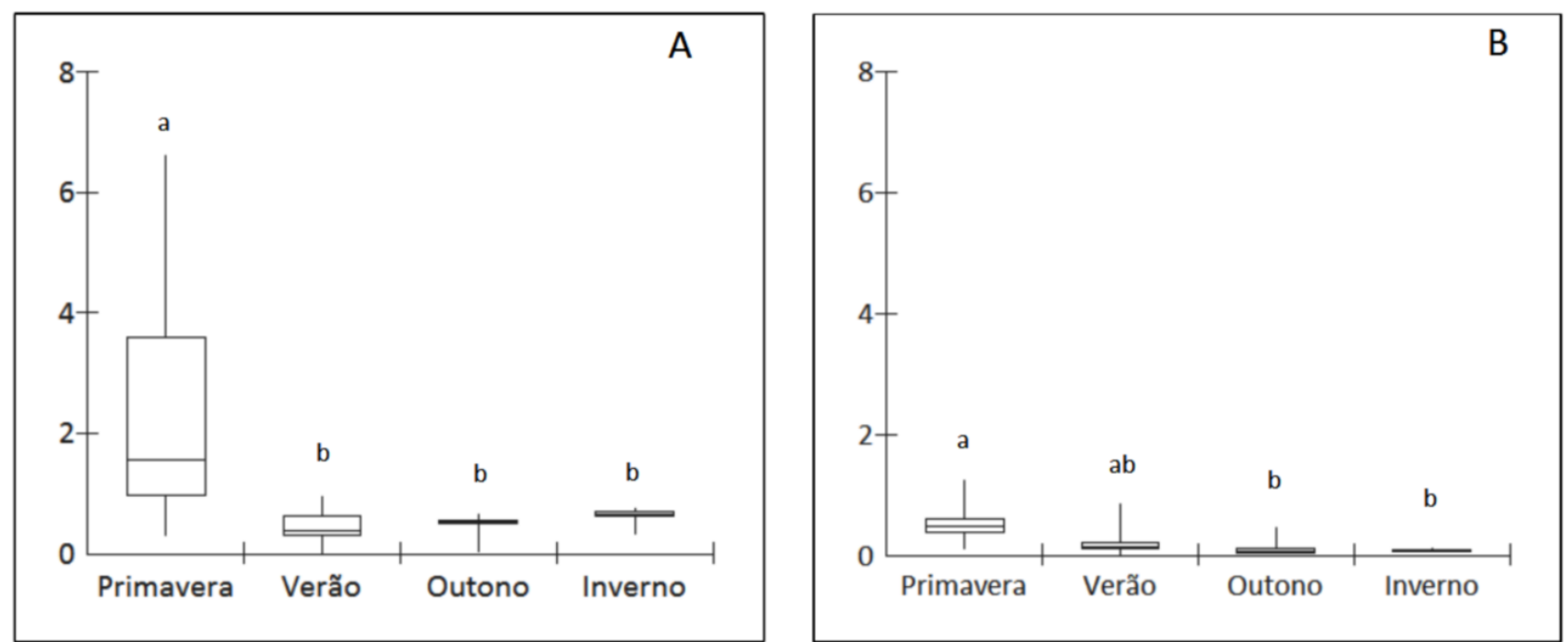

Figura 3. Medianas e desvios interquartílicos do índice gonadossomático (IGS) de fêmeas (A) e machos (B) de L. obtusidens coletados a jusante da UHE Funil, no município de Perdões (MG), no período de setembro/2006 a agosto/2007. Letras iguais indicam resultados significativamente iguais, letras diferentes, indicam valores significativamente diferentes, com $\mathrm{p}<0,05$.

Em relação à variação sazonal do índice hepatossomático, as fêmeas apresentaram os maiores valores $(\mathrm{p}<0,05)$ na primavera (Figura 4A).
Tendência semelhante foi observada nos machos, cujo IHS foi maior $(\mathrm{p}<0,05)$ na primavera, quando comparados com outono e inverno (Figura 4B). 

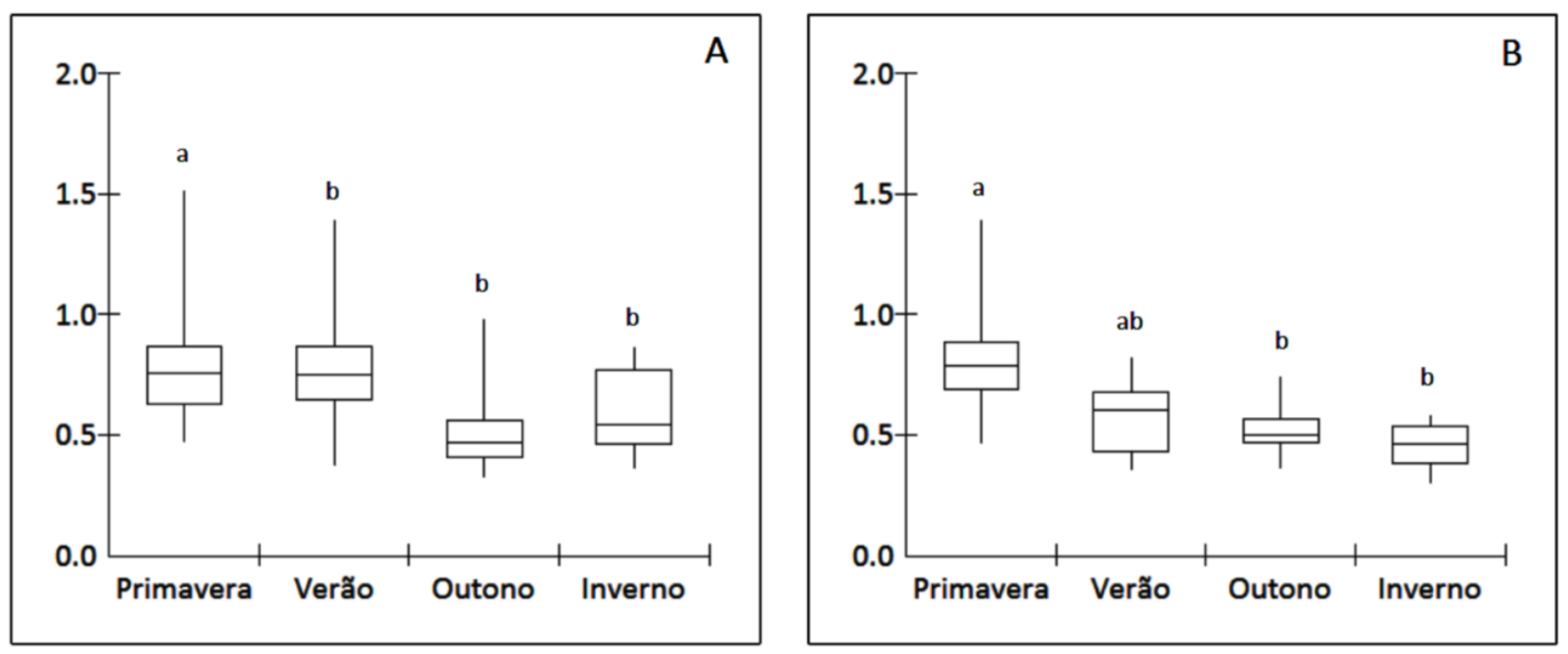

Figura 4. Medianas e desvios interquartílicos do índice hepatossomático (IHS) de fêmeas (A) e machos (B) de L obtusidens coletados a jusante da Usina Hidroelétrica do Funil, no município de Perdões (MG), no período de setembro/2006 a agosto/2007. Letras iguais indicam resultados significativamente iguais, letras diferentes, indicam valores significativamente diferentes, com $\mathrm{p}<0,05$.

Comparando-se as variáveis das fêmeas ao longo das estações, nota-se que existe correlação entre o K e IHS e entre IGS e IHS (Tabela 2).

Tabela 2. Coeficiente de correlação de Spearman comparando o Fator de Condição (K), Índice Gonadossomático (IGS) e Índice Hepatossomático (IHS) de L. obtusidens coletados a jusante da Usina Hidroelétrica do Funil, no município de Perdões (MG), no período de setembro/2006 a agosto/2007

\begin{tabular}{lcc}
\hline & $\mathrm{K}$ & IHS \\
\hline \multirow{2}{*}{ IHS } & $\mathrm{rs}=-0,2975$ & - \\
& $\mathrm{p}=0,02(*)$ & - \\
\hline \multirow{2}{*}{ IGS } & $\mathrm{rs}=-0,0251$ & $\mathrm{rs}=0,3881$ \\
& $\mathrm{p}=0,84(\mathrm{sn})$ & $\mathrm{p}=0,001\left(^{*}\right)$ \\
\hline *: correlacão altamente significativa &
\end{tabular}

*: correlação altamente significativa

sn: correlação não significativa

\section{DISCUSSÃO}

O valor do coeficiente $b=3,142$ encontrado na relação peso-comprimento de $L$. obtusidens situa-se dentro dos limites indicados por LE CREN (1951), que afirma que esses valores variam de 2,5 a 4,0 para a maioria das espécies de peixes. Esse coeficiente indica que a piapara apresentou crescimento alométrico positivo, ou seja, um maior incremento em peso do que em comprimento. $\mathrm{O}$ crescimento alométrico positivo, também foi observado em $L$. obtusinens coletadas após a formação do reservatório de Yacyretá, na Argentina (ARAYA et al., 2005).
Em peixes coletados em lagos da Amazônia, PRESTES et al. (2010) observaram crescimento alométrico negativo para três espécies de caraciformes (Triportheus albus, T. angulatus e $T$. auritus) e, segundo os autores, esse resultado é esperado para espécies com formato do corpo alongado e deprimido lateralmente. A relação pesocomprimento pode descrever o desenvolvimento relacionado aos estágios de vida de uma determinada espécie, mas a modificação das condições naturais, como por exemplo a formação de reservatórios, pode influenciar o tipo de crescimento (ARAYA et al., 2005).

O fator de condição (K) é um importante indicador do grau de higidez de um indivíduo e seu valor reflete as condições nutricionais recentes e/ou gastos das reservas em atividades cíclicas, sendo possível relacioná-lo às condições ambientais e aos aspectos comportamentais das espécies (GOMIERO et al., 2010). ANTONIUTTI et al. (1985) observaram que as maiores médias do $\mathrm{K}$ coincidem com a época de maturação gonadal do cascudo (Plecostomus albopunctatus) e BARBIERI \& VERANI (1987) chegaram às mesmas conclusões para outro locarídeo (Hypostomus aff. plecostomus).

Para o caraciforme $L$. obtusidens essa relação não foi possível, visto que o menor valor de $\mathrm{K}$ foi observado no verão, estação na qual ocorre sua desova (OLDANI et al., 1992), e pode refletir o gasto energético para a produção dos gametas. GOMIERO et al. (2010) também observaram o menor valor de K para o caraciforme Oligosarcus 
hepsetus durante o verão, estação que coincide com o período reprodutivo da espécie. Segundo os mesmo autores, os maiores valores de $\mathrm{K}$ no outono ocorreram devido à maior intensidade alimentar no final do inverno e primavera, com subsequente acúmulo de gordura no final do verão e outono (BOTELHO et al., 2007), podendo assim, sofrer alterações tanto em função dos fatores intrínsecos (reservas orgânicas, desenvolvimento gonadal e tamanho dos exemplares) quanto dos fatores extrínsecos, como temperatura, fotoperíodo e disponibilidade alimentar.

Dessa forma, a variação do $\mathrm{K}$ das piaparas também pode estar relacionada à disponibilidade e aproveitamento de alimentos pelos indivíduos, que pode ter sido maior durante o verão. LIMA-JÚNIOR \& GOITEIN (2006) observaram que, para o pimelodídeo $P$. maculatus, a variação sazonal do fator de condição não está diretamente relacionada ao desenvolvimento gonadal e que os indivíduos apresentaram maiores índices de condição corpórea após períodos de intensa atividade alimentar.

A hipótese da relação do $\mathrm{K}$ com fatores extrínsecos pode ser corroborada com resultado da correlação entre a variação sazonal do K e IGS, que pode indicar um maior desenvolvimento das gônadas. Nos testes de correlação, observou-se que essas duas variáveis apresentam ciclos independentes, ou seja, não há uma associação significativa entre elas.

Na primavera, foram observados os maiores valores de IGS, indicando que nessa época os animais encontraram condições ambientais e fisiológicas propícias à maturação gonadal, coincidindo com a elevação das médias pluviométricas e da média de temperatura (SANTOS et al., 2006). Dessa forma, com a análise do IGS de machos e fêmeas, pode-se inferir que o período reprodutivo de $L$. obtusidens, no local estudado, pode estar compreendido durante a primavera.

O IHS também pode ser utilizado como indicador do período reprodutivo de peixes, correlacionado a outros fatores como o $\mathrm{K}$ e IGS. Esse índice pode estar relacionado com a mobilização das reservas energéticas necessárias para o processo de vitelogênese, reprodução ou também para preparação para o período de inverno (QUEROL et al., 2002).

Para L. obtusidens capturadas na barragem do Funil, o IHS pode estar relacionado com o período reprodutivo dessa espécie. Os menores valores do IHS de fêmeas foram observados no outono e inverno e podem ser decorrentes do gasto energético que a espécie sofreu durante o período reprodutivo (primavera). Nos machos, o IHS também foi menor no outono, indicando que as reservas energéticas podem ter sido utilizadas para a reprodução. Esse fato pode ser evidenciado pela existência de correlação altamente significativa entre o IHS e o IGS.

Em contrapartida, QUEROL et al. (2002) concluiram que o IHS do cascudo (Loricariichthys platymetopon) não pode ser utilizado como indicador do período reprodutivo, pois apresenta seus maiores índices no outono. Segundo os mesmo autores, o IHS dos cascudos pode estar relacionado com o acúmulo de reservas energéticas para o período de inverno e não ao período de reprodução. Esse fato foi evidenciado pelos autores pela baixa correlação entre o IHS e o IGS $(-0,21)$.

\section{CONCLUSÃO}

Com a análise do IGS de fêmeas e machos, pode-se inferir que o período reprodutivo de $L$. obtusidens, capturadas a jusante da Usina Hidroelétrica do Funil, ocorre durante a primavera. Relacionados, os índices IGS e IHS podem ser utilizados como indicadores reprodutivos, enquanto que o fator de condição (K) não foi um bom indicador do período reprodutivo para essa espécie.

\section{REFERÊNCIAS}

AGOSTINHO, A. A.; BARBIERI, G.; VERANI, J. R.; HAHN, N. S. Variação do fator de condição e do índice hepatossomático e suas relações com o ciclo reprodutivo em Rhinelepis aspera (Agassis, 1829) (Osteichthyes, Loricariidae) no Rio Paranapanema, Porecatu. Ciência e Cultura, v. 42, p. 711-714, 1990.

AGOSTINHO, A. A.; GOMES, L. C.; PELICICE, F. M. Ecologia e manejo de recursos pesqueiros em reservatórios do Brasil. Maringá. 2007. Eduem, 501 p.

AGOSTINHO, A. A.; ZALEWSKI, M. The dependence of fish community structure and dynamics on floodplain and riparian ecotone zone in in Parana River, Brazil. Hydrobiologia, v. 303, p. 141-148, 1995.

ANTONIUTTI, D. M.; RANZANI-PAIVA, M. J. T.; GODINHO, H. M. Morfologia das gônadas, escala de maturidade e fator de condição de Plecostomus albopunctatus Regan, 1908 (Osteichthyes, Loricariidae) do rio Jaguari, São Paulo, Brasil. Boletim do Instituto de Pesca, v. 12, p. 87-103, 1985.

ARAYA, P. R.; AGOSTINHO, A. A.; BECHARA, J. A. The influence of dam construction on a population of Leporinus obtusidens (Valenciennes, 1847) (Pisces, Anostomidae) in the Yacyreta Reservoir (Argentina). Fisheries Research, v. 74, p. 198-209, 2005.

BARBIERI, G.; VERANI, J. R. O fator de condição como indicador do período de desova em Hypostomus aff. 
plecostomus (Linnaeus, 1758) (Osteichthyes, Loricariidae), na represa do Monjolinho (São Carlos, SP). Ciência e Cultura, v. 39, p. 655-658, 1987.

BOTELHO, M. L. L. A., GOMIERO, L. M.; BRAGA, F. M. S. 2007. Feeding of Oligosarcus hepsetus (Cuvier, 1829) (Characiformes) in the Serra do Mar State Park Santa Virgínia Unit, São Paulo, Brazil. Brazilian Jouranl of Biology, v. 67, n. 4, p. 741-748, 2007.

GOMIERO, L. M.; VILLARES-JUNIOR, G. A.; BRAGA F. M. S. Relação peso-comprimento e fator de condição de Oligosarcus hepsetus (Cuvier, 1829) no Parque Estadual da Serra do Mar - Núcleo Santa Virgínia, Mata Atlântica, estado de São Paulo, Brasil. Biota Neotropica, v. 10, n. 1, p. 101-105. 2010.

GOMIERO, L. M.; GARUANA, L.; BRAGA, F. M. S. Reproduction of Oligosarcus hepsetus (Cuvier, 1829) (Characiformes) in the Serra do Mar State Park, São Paulo, Brazil. Brazilian Journal of Biology, v. 68, n. 1, p. 187-192, 2008.

LE CREN, E. D. The lenght-weight relationship and seasonal cycle in gonad weight and condition in the perch (Perca fluviatilis). Journal of Animal Ecology, n. 20, p. 201-219, 1951.

LIMA-JUNIOR, S. E.; CARDONE. I. B.; GOITEIN, R. Determination of a method for calculation of Allometric Condition Factor of fish. Acta Scientiarium, v. 24, n. 2, p. 397-400, 2002.

LIMA-JUNIOR, S. E.; GOITEIN, R. Fator de condição e ciclo gonadal de fêmeas de Pimelodus maculatus (Osteichthyes, Pimelodidae) no rio Piracicaba (SP, Brasil). Boletim do Instituto de Pesca, v. 32, n. 1, p. 87-94, 2006.

NETO, J. R.; LAZZARI, R.; PEDRON, F. R.; VEIVERBERG, C. A.; BERGAMIN, G. T.; CORRÊIA, V.; FILIPETTO, J. E. S. Alimentação da piava (Leporinus obtusidens) com diferentes fontes protéicas. Ciência
Rural, v. 36, p. 1611-1616, 2006.

OLDANI, N.; IWASZKIW, J.; PADÍN, O.; OTAEGUI, A. Fluctuaciones de la abundancia de peces en el Alto Paraná (Corrientes, Argentina). Publicaciones de la Comisión Administradora del Río Uruguay, Série Técnico-Científica, v. 1, p. 43-55, 1992.

PRESTES, L., SOARES, M.G.M., SILVA, F.R; BITTENCOURT, M.M. Dinâmica populacional de Triportheus albus, T. angulatus e $T$. auritus (CHARACIFORMES: CHARACIDAE) em lagos da Amazônia Central. Biota Neotropica, v. 10, n. 3, 2010.

QUEROL, M. V. M.; QUEROL, E.; GOMES, N. N. A. Fator de condição gonadal, índice hepatossomático e recrutamento como indicadores do período de reprodução de Loricariichthys platymetopon (Osteichthyes, Loricariidae), Bacia do Rio Uruguai Médio, Sul do Brasil. Iheringia, Série Zoologia, v. 92, n. 3, p. 79-84, 2002.

SANTOS, S. L.; VIANA, L. F.; LIMA-JUNIOR, S. E. Fator de condição e aspectos reprodutivos de fêmeas de Pimelodella cf. gracilis (Osteichthyes, Silutiformes, Pimelodidae) no rio Amambai, Estado de Mato Grosso do Sul. Acta Scientiarium Biological Sciences, v. 28, n. 2, p. 129-134, 2006.

SUZUKI, H. I.; AGOSTINHO, A. A. Reprodução de peixes do reservatório de Segredo. In: AGOSTINHO, A. A.; GOMES, L. C. Reservatório de Segredo, bases ecológicas para o manejo. Maringá. Eduem. p. 163-182, 1997.

TAITSON, P. F.; CHAMI, E.; GODINHO, H. P. Gene banking of the neotropical fish Leporinus obtusidens (Valenciennes, 1836): a protocol to freeze its sperm in the field. Journal of Animal Science, v. 105, p. 283-291, 2008.

VAZZOLER, A. E. A. M. Biologia da reprodução de peixes teleósteos: teoria e prática. Maringá: NUPELIA, 169 p. 1996. 